\title{
Evaluación Morfológica y Variaciones del Circulo Arterial Cerebral. Un Estudio con Material Cadavérico en una Muestra de Población Colombianas
}

\author{
Morphological Evaluation and Variations of the Cerebral Arterial Circle. \\ A Study with Cadaveric Material in a Colombian Population Sample
}

Luis Ernesto Ballesteros Acuña ${ }^{1}$; Natalia García Corredor ${ }^{2}$ \& Oscar Javier Larrotta Rojas ${ }^{2}$

\begin{abstract}
BALLESTEROS, A. L. E.; GARCÍA, C. N. \& LARROTTA, R. O. J. Evaluación morfológica y variaciones del circulo arterial cerebral . Un estudio con material cadavérico en una muestra de población colombiana. Int. J. Morphol., 39(5):1453-1458, 2021.

RESUMEN: El círculo arterial cerebral (CAC) mencionado también como polígono de Willis es una red de anastomosis vascular situado en la base del cerebro, constituido por ramas de la arteria carótida interna (ACI) y del sistema vertebro-basilar. Este estudio evaluó la morfología de las arterias que conforman el CAC en una muestra de individuos colombianos. Previa canalización de la ACI y de la arteria vertebral (AV), se perfundió con resina poliéster (palatal $85 \%$ y estireno $15 \%$ ) los lechos vasculares del CAC de 70 encéfalos extraídos de cadáveres a quienes se les practicó necropsia en el Instituto de Medicina Legal de Bucaramanga, Colombia. La arteria comunicante anterior (ACoA) se observó en 68 encéfalos $(97,1 \%)$, con ausencia en 2 de las muestras (2,9\%); en promedio su

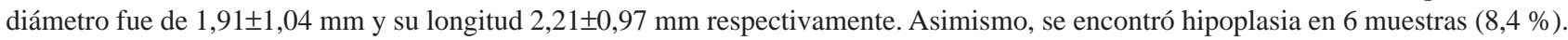
La arteria comunicante posterior (ACoP) estuvo ausente en 2 de las muestras (5,7 \%); su longitud fue 11,63 $\pm 2,12 \mathrm{~mm}$, mientras que su calibre fue de 1,21 $\pm 0,58 \mathrm{~mm}$, siendo ligeramente mayor en el lado derecho, sin diferencias estadísticamente significativas con relación al lado de presentación ( $\mathrm{p}=0,763)$. Se encontró hipoplasia de la ACoP en forma bilateral en 19 de las muestras $(27,1 \%)$ y unilateral en 15 muestras $(21,4 \%)$. En 8 muestras $(20 \%)$ de 35 CAC evaluados se observó configuración fetal. La incidencia de hipoplasia de la ACoP y de configuración fetal encontrados en el presente estudio, se ubican en el segmento superior de lo reportado en la literatura. Estas expresiones morfológicas han sido consideradas como coadyuvantes en el desarrollo de accidentes cerebro-vasculares (ACV).
\end{abstract}

PALABRAS CLAVE: Círculo arterial cerebral;Arteria cerebral posterior;Arteria comunicante anterior; Arteria comunicante posterior; Variaciones anatómicas.

\section{INTRODUCCIÓN}

El sistema de irrigación cerebral está conformado por una red arterial anastomosada denominada círculo arterial cerebral (CAC), que tiene como función importante el suministro del flujo sanguíneo y transporte de nutrientes al encéfalo. El CAC (polígono de Willis), está conformado por el aporte de las dos arterias carótidas internas (ACI) y de la arteria basilar (AB). Ubicándose en la base del cerebro y rodeando al nervio, quiasma, tractos ópticos, hipófisis y la parte inferior del hipotálamo. Situado en el espacio subaracnoideo donde surgen las ramas que irrigan a los hemisferios cerebrales. (Raghavendra et al., 2014). Se le identifica un segmento vascular anterior derivado de las ACI y uno posterior o vertebro basilar. Su segmento anterior está constituido por las porciones proximales de las arterias cerebrales anteriores derecha e izquierda unidas entre sí por la arteria comunicante anterior (ACoA); mientras que su segmento posterior lo constituye la porción proximal de las arterias cerebrales posteriores derecha e izquierda provenientes de la $\mathrm{AB}$, unidas a las $\mathrm{ACI}$ por medio de las arterias comunicantes posteriores (ACoP). EL CAC es considerado con suma frecuencia como bilateralmente simétrico, manteniendo de esa manera una equilibrada perfusión cerebral (Siddiqi et al., 2013; Raghavendra et al.).

Variantes anatómicas de la configuración del CAC han sido reportadas en un rango del 20-50 \% por medio de diferentes técnicas de identificación, como estudios con material cadavérico, tomografía angiográfica computarizada,y la resonancia angiográfica magnética que muestran las características hemodinámicas del CAC que

\footnotetext{
${ }^{1}$ Morfología, Universidad Industrial de Santander, Bucaramanga, Colombia.

${ }^{2}$ Ciencias Básicas Biomédicas, Universidad Industrial de Santander, Bucaramanga, Colombia.
} 
pueden determinar disminución o incremento de la perfusión cerebral (Hoksbergen et al., 2003; De Silva et al., 2011; Hernández-Luna et al., 2015).

La definición y descripción de hipoplasia para la ACoA y la ACoP, es cuando estos vasos presentan un diámetro externo menor a $1 \mathrm{~mm}$ (Puchades-Orts et al., 1976; Siddiqi et al.). Para la ACoP hay reportes de hipoplasia entre el 25-40\%, mientras que para la ACoAdel14-40\% (Puchades-Orts et al.; Mandiola et al., 2005; Siddiqi et al.; Raghavendra et al.; El Falougy et al., 2018). Las duplicaciones y agenesias de estructuras vasculares del CAC se han relacionado con alteraciones funcionales cerebrales (Hoksbergen et al.; Mandiola et al.). Entre las variables cualitativas relevantes destacan la ACoP fenestrada 0,5-10\% y con agenesia entre el 1,5-18\% (Siddiqi et al.; Raghavendra et al.; Gunnal et al., 2018). Valores de agenesia de ACoA se reportan entre 2-10\% (Radhavendra et al.; Gunnal et al.).

Diversos estudios han reportado las configuraciones del segmento posterior del CAC tomando en cuenta las variaciones de su diámetro, específicamente de la ACoP (De Silva et al., 2009; Gunnal et al.; Wijesinghe et al., 2020). Se han descrito tres patrones: en el patrón adulto el segmento precomunicante (P1) de la arteria cerebral posterior (ACP) tiene un diámetro mayor en comparación a la ACoP (43-80 \%);-en el patrón transicional el diámetro de la ACoP es igual al de P1(7- $47 \%)$, mientras que en el patrón fetal el diámetro del $\mathrm{P} 1$ es menor que el de la ACoP (13-16 \%) (El Falougy et al.; Wijesinghe et al.).

Según sea el patrón del segmento posterior del CAC, se generan diferentes efectos de suplencia de flujo sanguíneo hacia las diferentes áreas corticales y del diencéfalo; y es proclive a que se presenten o en su efecto se eviten procesos patológicos importantes, debido a que la ACoP permite la comunicación con el segmento anterior del CAC (Gunnal et al.).

Las arterias que conforman el CAC han sido ampliamente estudiadas por diversos autores, (Sidiqqui et al., 2013; De Silva et al., 2011; El Falougy et al.); sin embargo, en población colombiana, son escasos los estudios anatómicos con material cadavérico que profundicen sobre las expresiones morfológicas del CAC, con relación a la simetría, variaciones anatómicas biométricas y patrones cualitativos. Considerando la variabilidad anatómica del CAC y sus correspondientes implicaciones clínicas-quirúrgicas, este estudio evaluó la morfología del CAC en una muestra cadavérica de población colombiana mestiza con el fin de obtener información primordial y propia de referencia en la región.

\section{MATERIAL Y MÉTODO}

Este estudio descriptivo valoró las características anatómicas del CAC de 70 encéfalos extraídos de cadáveres frescos de individuos a quienes se les Practicó necropsia en el Instituto de Medicina Legal de Bucaramanga-Colombia-, que no presentaban traumatismo craneoencefálico contuso y/o penetrante. Adicionalmente, para la selección del material de estudio se tuvieron en cuenta los siguientes criterios de inclusión: sujetos masculinos mestizos de 2080 años (no se evaluaron casos femeninos en razón al escaso número de autopsias forenses practicadas a mujeres) y sin patologías agudas o crónicas que comprometieran al sistema nervioso central.

A cada espécimen cadavérico se le practicó el siguiente procedimiento: incisión en " $\mathrm{V}$ " desde el proceso mastoideo hasta la incisura yugular, con compromiso de piel y tejido celular subcutáneo, disección bilateral por planos de las regiones anterior y lateral del cuello, con exposición del componente muscular, seguido de desinserción de los músculos esternocleidomastoideos al nivel de su origen con reclina miento de las clavículas. Posteriormente, se individualizo a las estructuras del paquete neurovascular del cuello y disecó la arteria vertebral (AV).

Luego, se procedió a canalización bilateral de los segmentos proximales de ACI y AV. A través de éstas, se practicó lavado de los lechos vasculares con suero fisiológico y prefijado del encéfalo con formaldehido al $3 \%$. Posteriormente, se perfundió $100 \mathrm{cc} 3$ de resina semisintética (mezcla de palatal E210® Basf 80 cc3estireno $20 \mathrm{cc} 3$ ) impregnada de color rojo mineral. Una vez obtenida la polimerización de la resina (30 minutos), se practicó la exéresis del encéfalo. A continuación, la muestra se sometió a fijación con formaldehido al $10 \%$ durante 10 días.

Obtenida la fijación del encéfalo, se practicó limpieza de las leptomeninges, maniobra que permitió identificar plenamente cada una de las estructuras constituyentes del CAC. Mediante la utilización de calibrador digital (Mitotuyo ${ }^{\circledR}$ ). Se realizó la medición de la longitud y diámetro externo de cada una de las estructuras del CAC. Se señalaron los casos de agenesia para A1, ACoA, ACoP y P1 y de hipoplasia de estos vasos, con calibre menor de un milímetro (Puchades-Orts et al.) y la trayectoria horizontal, oblicua, duplicada y fenestrada de la ACoA (De Silva et al., 2009). Igualmente, se registró las expresiones adulta, transicional y fetal del CAC. Los hallazgos de cada una de las muestras fueron fotografiados, con cámara digital (Cannon T2I). Los datos obtenidos fueron registrados y almacenados en Microsoft Excel con procesamiento ana- 
lítico a través del software estadístico SigmaStat 3.5. Las variables continuas se describieron con promedios y desviación estándar; las variables nominales con sus proporciones. Se realizo t-test como prueba de hipótesis, aceptando un nivel de significancia de $\mathrm{p} \leq 0,05$.

\section{RESULTADOS}

Se estudiaron las estructuras vasculares del CAC de 70 encéfalos, de sujetos masculinos de 18-65 años, con edad media de 33,8 años. Para la ACoA, se observó en 68 encéfalos $(97,1 \%)$ mientras que en 2 muestras $(2,9 \%)$ estuvo ausente; con un diámetro promedio de 1,91 $\pm 1,04$ $\mathrm{mm}$ y con hallazgos de hipoplasia en 6 de las muestras $(8,4 \%)$. Así mismo, su longitud fue de $2,21 \pm 0,97 \mathrm{~mm}$. La ACoA, presentó una trayectoria transversa (tipo 1; Figs. $1 \mathrm{~A}, \mathrm{~B})$ en 40 muestras $(58,8 \%)$ con un diámetro $2,04 \pm 0,94$ $\mathrm{mm}$ y longitud de $1,92 \pm 0,88 \mathrm{~mm}$, seguido de la trayectoria oblicua derecha a izquierda (tipo 2) en 13 muestras $(19,1 \%)$ con diámetro de $1,73 \pm 0,7 \mathrm{~mm}$ (Tabla I).

El segmento A1 de las ACA presentó un calibre bilateral de 2,36 $\pm 0,38 \mathrm{~mm}$. La longitud del segmento A1 derecho fue de $12.78 \pm 1,48 \mathrm{~mm}$ mientras que en el hemisferio contralateral fue de 12,27 $\pm 1,65 \mathrm{~mm}$, sin diferencias estadísticamente significativas según lateralidad $(\mathrm{p}=0,085)$. La ACoP estuvo ausente en 2 de las muestras $(5,71 \%)$, con una longitud promedio de $11,63 \pm 2,12 \mathrm{~mm}$, mientras que su calibre fue de $1,21 \pm 0,58 \mathrm{~mm}$, siendo ligeramente mayor en el lado derecho $(p=0,763)$. Se encontró hipoplasia de la ACoP de forma bilateral, en 19 de las muestras $(27,1 \%)$ y unilateral en 15 especímenes $(21,4$ $\%)$; con una hipoplasia unilateral derecha en el $10 \%$ de los casos y $11,4 \%$ unilateral izquierda.

El segmento P1 de las arterias cerebral posterior presentó una longitud de $6,3 \pm 1,77 \mathrm{~mm}$, con un diámetro para $\mathrm{P} 1$ en el lado derecho de $2,30 \pm 0,51 \mathrm{~mm}$ y del lado izquierdo de 2,36 $\pm 0,43 \mathrm{~mm},(\mathrm{p}=0,556)$ (Tabla II). Se encontró además hipoplasia bilateral en una muestra $(1,4 \%)$. De las variaciones anatómicas cualitativas del CAC se encontró ACoA única en 64 muestras $(91,2 \%)$ y duplicada en 6 muestras $(8,8 \%)$ (Fig. 2). Se registro la configuración fetal, transicional y adulta del segmento posterior de CAC (Fig. 2) en 35 muestras, con 2 muestras de circulación fetal bilateral $(5,7 \%$ ) (Fig. 3), unilateral derecho en 3 muestras $(8,6 \%)$ y unilateral izquierdo en 2 muestras $(5,7$ $\%)$ (Tabla III).
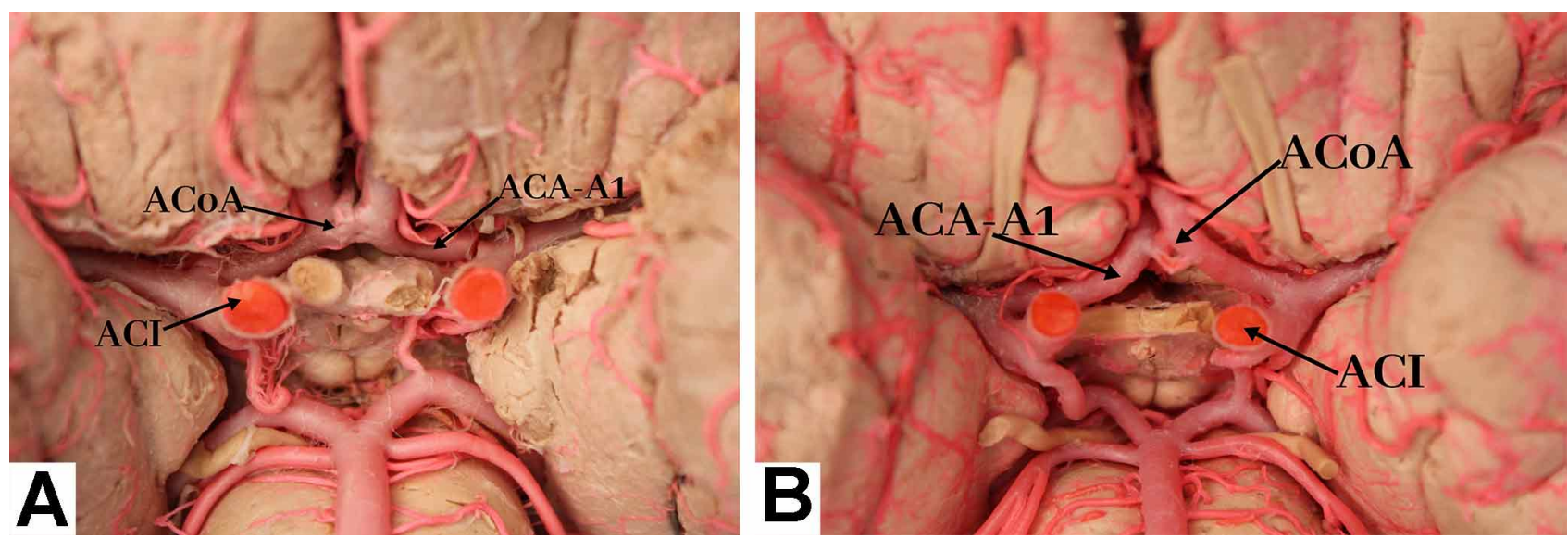

Fig. 1. A. Trayectoria transversa Tipo 1 de la arteria comunicante anterior. ACA-A1: segmento precomunicante de la arteria cerebral anterior. ACI: arteria carótida interna. ACoA: arteria comunicante anterior. B. Trayectoria fenestrada Tipo 4 de la arteria comunicante anterior. ACA-A1: segmento precomunicante de la arteria cerebral anterior. ACI: arteria carótida interna. ACoA: arteria comunicante anterior. Además, se observa configuración Adulta unilateral izquierda y transicional derecha.

Tabla I. Valor promedio y desviación estándar (mm) del diámetro y longitud de la arteria comunicante anterior (ACoA) según el tipo de configuración.

\begin{tabular}{|c|c|c|c|c|c|c|}
\hline & \multicolumn{5}{|c|}{$\mathrm{ACoA}$} & \multirow[b]{2}{*}{ Total } \\
\hline & $\begin{array}{c}\text { Tipo } 1 \\
\text { Transversa }\end{array}$ & $\begin{array}{l}\text { Tipo } 2 \\
\text { Oblicu a derecha }\end{array}$ & $\begin{array}{l}\text { Tipo } 3 \\
\text { Oblicua izquierda }\end{array}$ & Tipo 4 fenestrada & $\begin{array}{c}\text { Tipo } 5 \\
\text { Fusionada }\end{array}$ & \\
\hline No $(\%)$ & $40(58,8 \%)$ & $13(19,1)$ & $8(11,8)$ & $4(5,9)$ & $3(4,4 \%)$ & $68(100)$ \\
\hline Diámetro & $2,04 \pm 0,94$ & $1,73 \pm 0,7$ & $1,4 \pm 1,1$ & $1,61 \pm 0,64$ & $3,33 \pm 1,88$ & $1,91 \pm 1,04$ \\
\hline Longitud & $1,92 \pm 0,88$ & $2,54 \pm 1,05$ & $2,4 \pm 0,8$ & $2,81 \pm 1,51$ & $3,86 \pm 0,46$ & $2,21 \pm 0,97$ \\
\hline
\end{tabular}




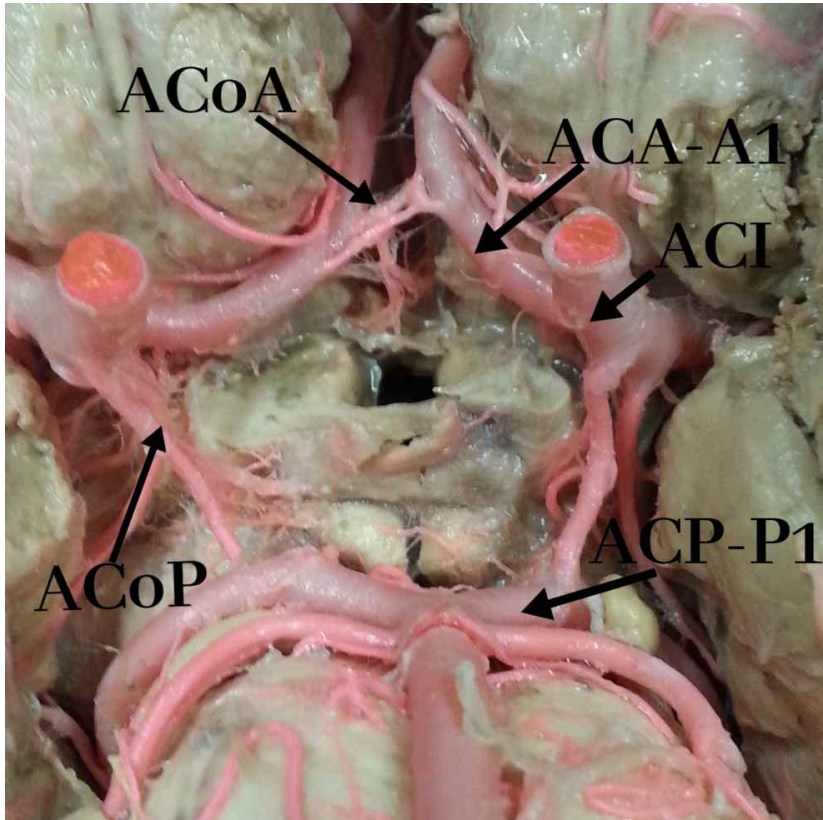

Fig. 2. Trayectoria doble y oblicua izquierda de la arteria comunicante anterior y configuración adulta bilateral del segmento posterior del Circulo arterial del cerebro. ACA-A1: segmento precomunicante de la arteria cerebral anterior. ACI: arteria carótida interna. ACoA: arteria comunicante anterior. ACoP: arteria comunicante posterior. ACP-P1: segmento precomunicante de la arteria cerebral posterior.

Tabla II. Valor promedio y desviación estándar (mm) del diámetro y longitud del segmento posterior del círculo arterial cerebral, según el lado de presentación.

\begin{tabular}{lccccc}
\hline Arteria & & Derecho & Izquierdo & Total & P \\
\hline ACP & Diámetro & $2,30 \pm 0,51$ & $2,36 \pm 0,43$ & $2,33 \pm 0,47$ & 0,556 \\
(P1) & Longitud & $6,53 \pm 1,95$ & $6,02 \pm 1,59$ & $6,3 \pm 1,77$ & 0,195 \\
ACoP & Diámetro & $1,24 \pm 0,61$ & $1,18 \pm 0,55$ & $1,21 \pm 0,58$ & 0,763 \\
& Longitud & $11,66 \pm 2,44$ & $11,60 \pm 1,81$ & $11,63 \pm 2,12$ & 0,985 \\
\hline
\end{tabular}

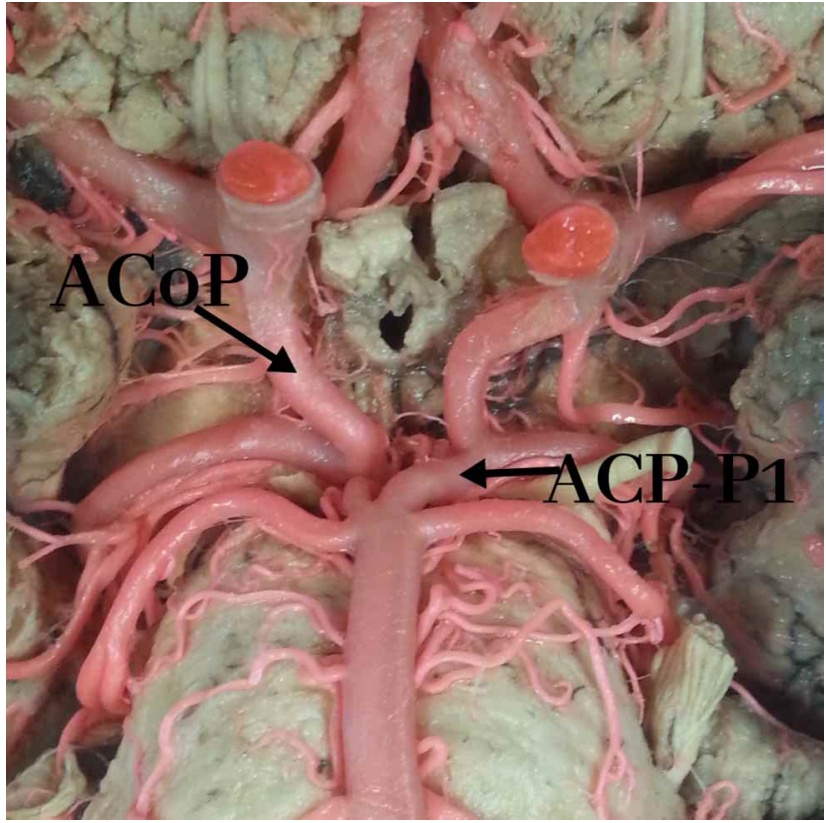

Fig. 3. Configuración fetal bilateral del segmento posterior del circulo arterial del cerebro. ACoP: arteria comunicante posterior. ACP$\mathrm{P} 1$ : segmento precomunicante de la arteria cerebral posterior.

Tabla III. Configuración adulta, transicional y fetal del circulo arterial del cerebro.

\begin{tabular}{lccc}
\hline \multicolumn{2}{l}{ Configuración } & $\begin{array}{c}\mathrm{N}=35 \\
\text { No }\end{array}$ & $\%$ \\
\hline Adulta & & & \\
\multirow{7}{*}{ Fetal } & Bilateral & 27 & 77,1 \\
& & & \\
& Bilateral & 2 & 5,7 \\
& Izquierda & 2 & 5,7 \\
$\quad$ Derecha & 3 & 8,6 \\
Transicional & & \\
Bilateral & - & - \\
& Izquier da & - & - \\
Derecha & 1 & 2,9 \\
\hline
\end{tabular}

\section{DISCUSIÓN}

El CAC presenta gran variabilidad en su configuración y morfometría, expresada específicamente en el calibre, longitud, ausencias y trayectorias de las estructuras que lo constituyen (Mandiola et al.; Sidiqqui et al.; De Silva et al., 2011; El Falougy et al.).

La longitud y calibre de la ACoA registrada en este estudio (2,21 mm; 1,91 mm), es similar a hallazgos encontrados en la literatura (Mandiola et al.; Raghavendra et al.). Para este vaso se ha reportado una duplicación entre 4-33 $\%$; nuestros hallazgos $(8,8 \%)$ son concordantes con lo reportado por Mandiola et al. y es menor al $16 \%$ reportado por Pacheco Mayedo et al. (2017). Se destaca la incidencia de 33,3\% señalada por Gomes et al. (1986). La agenesia de la ACoA es reportada en la literatura en un rango de 3-22,8 $\%$ (Raghavendra et al.; Monroy-Sosa et al., 2013). La agenesia de este vaso, observada en nuestro estudio $(2,9 \%)$ es similar a lo reportado por Monroy-Sosa et al.

La longitud de la ACoP reportada por Gunnal et al. de 15,3 mm, es mayor a los resultados del presente estudio. Nuestros hallazgos son concordantes con los reportados por El Falougy et al. (10,62 mm hemisferio derecho y 10,48 $\mathrm{mm}$ hemisferio izquierdo). Así mismo, nuestros registros relacionados conel diámetro, se ubican en el segmento inferior del rango de 1,1-2 $\mathrm{mm}$ reportado en estudios previos (Gunnal et al.; El Falougy et al.). 
BALLESTEROS, A. L. E.; GARCÍA, C. N. \& LARROTTA, R. O. J. Evaluación morfológica y variaciones del circulo arterial cerebral . Un estudio con material cadavérico en una muestra de población colombiana. Int. J. Morphol., 39(5):1453-1458, 2021.

Tabla IV. Ausencia e hipoplasia de la arteria comunicante posterior y patrones fetal, adulto y transicional del circulo arterial del cerebro. Según autores.

\begin{tabular}{|c|c|c|c|c|c|c|}
\hline Autor & Ausencia & \multicolumn{2}{|c|}{ Hipoplasia } & \multicolumn{3}{|c|}{ Patrón } \\
\hline & & UNI & BIL & Fetal & Adulto & Transicional \\
\hline Gunnal et al. (2018) & 3,5 & & 25,3 & 16,4 & 79,4 & - \\
\hline Raghavendra et al. (2014) & 18,2 & -- & 31,8 & -- & -- & -- \\
\hline E1 Falougy et al. (2018) & 1,6 & 17,6 & 16,7 & 13,2 & 43,2 & 7,4 \\
\hline De Silva et al. (2009) & -- & 11,5 & 23 & 4,4 & 93,3 & 2,2 \\
\hline Sidiqqui, et al. (2013) & 15,7 & 39,5 & -- & -- & -- & -- \\
\hline Saeki and Rhoton. (1977) & -- & -- & 32 & 22 & 54 & -- \\
\hline Presente estudio. (2021). & 5,7 & 21,4 & 27,1 & 20 & 77,1 & 2,9 \\
\hline
\end{tabular}

El calibre $(2,36 \pm 0,38 \mathrm{~mm})$ y longitud $(12,78 \pm 1,48$ $\mathrm{mm})$ del segmento A1 encontrados en nuestra serie son concordantes con los reportes de Mandiola et al. y Kedia et al. (2013). La hipoplasia de la ACoA observada en este estudio $(8,4 \%)$, es considerablemente más baja que la reportada en estudios previos (Mandiola et al.;Sidiqqui et al.). Mientras que la hipoplasia de ACoP se ha reportado en un rango de $17-53 \%$, con predominio del escenario unilateral sobre el bilateral y con dominancia derecha (Sidiqqui et al.; De Silva et al., 2011; El Falougy et al.).Nuestros hallazgos se ubican en el segmento superior de los reportes de esta característica en la literatura (Tabla IV).

La configuración fetal de la ACoP se ha reportado entre el 13-30 \% (Saeki \& Rhoton Jr., 1977; El Falougy et al.; Gunnal et al.; Wijesinghe et al.). En este estudio la expresión fetal $(20 \%)$ se ubica en el segmento superior a lo reportado en la literatura y concordante con los reportes Saeki \& Rhoton Jr. El calibre del segmento P1 encontrado (2,30 \pm 0.38 derecho; $2,36 \pm 0,43$ izquierdo), son concordantes con los reportes de Kamath (1981), y considerablemente mayores a los datos de Krishnamurthy et al. (2008). La longitud de P1en nuestra serie con $6,53 \mathrm{~mm}$ del hemisferio derecho y $6,02 \mathrm{~mm}$ del lado contralateral se encuentra dentro del rango $(6,8-7,5 \mathrm{~mm})$ de lo reportado por Kamath y Krishnamurthy et al.

Se han propuesto varias teorías para explicar las anomalías y variaciones del CAC como las que señalan que probablemente están determinadas genéticamente y se desarrollan en la etapa embrionaria temprana y persisten en la vida posnatal a los que podrían sumarse factores bioquímicos y físicos aleatorios (Milenkovic et al., 1985). En este sentido, Van Overbeeke et al. (1991) observaron que las variaciones en los segmentos posteriores del círculo se debían a modificaciones del desarrollo con mayores demandas funcionales en relación con el rápido crecimiento de los lóbulos occipitales. También se ha señalado como factores determinantes de esta variabilidad a los delicados ajustes hemodinámico de todos los segmentos del CAC y a los cambios de flujo en las arterias aferentes durante los movimientos del cuello a lo largo de la vida.
Los cambios en la configuración de la ACoP y la agenesia de algunas estructuras del CAC, puede resultar en riesgo para el desarrollo de accidentes cerebrovasculares (Kapoor et al., 2008). Así mismo, se conoce que la función colateral del CAC es relevante en pacientes con oclusiones y patologías de la ACI teniendo en cuenta que, al presentarse una enfermedad obstructiva de un sistema ipsilateral, el sistema contralateral contribuirá a suplir flujo sanguíneo a través de la ACoP (Hoksbergen et al.; Wijesinghe et al.). La capacidad colateral del CAC fue evaluada por Hoksbergen et al. mediante el uso de ecografía dúplex transcraneal, en pacientes ateroscleróticos sin síntomas cerebro vasculares y concluyeron que la vía colateral anterior (ACoA) del círculo era siempre funcional, mientras que la vía colateral posterior (PCoA) no es funcional en casi la mitad de los hemisferios.

Se ha postulado que las variaciones anatómicas de los vasos del CAC son un factor de riesgo involucrado con las enfermedades cardiovasculares y en la progresión y ruptura de las lesiones aneurismáticas, por lo que estudios quirúrgicos, imagenológicos, y de autopsia han establecido su asociación (Martínez et al., 2004; Ingebrigtsen et al., 2004), pero la incidencia de éstas se comporta de manera diferente según la población, etnia y grupo racial (Eftekhar et al., 2006; Kapoor et al.). De igual manera, se ha correlacionado la configuración asimétrica del CAC como factor etiopatogénico de aneurismas ubicados principalmente en el origen de la ACoA (Charbel et al., 1991). Se ha establecido que la dominancia de flujo derecha o izquierda resultante de esta asimetría, puede ser un factor importante en el origen y desarrollo de estas lesiones.

Este estudio, realizado en material cadavérico, brinda información cualitativa y biométrica relevante del CAC. Los hallazgos obtenidos de estas estructuras vasculares enriquecen el concepto morfológico, permitiendo optimizar las intervenciones quirúrgicas, con el fin de prevenir y / o reducir déficits y daño neurológico postoperatorio, así como el diagnóstico y manejo de eventos clínicos que comprometan a los componentes del CAC. 
BALLESTEROS, A. L. E.; GARCÍA, C. N. \& LARROTTA, R. O. J. Morphological evaluation and variations of the cerebral arterial circle. A study with cadaveric material in a Colombian population sample. Int. J. Morphol., 39(5):1453-1458, 2021.

SUMMARY: The cerebral arterial circle (CAC), also referred to as the polygon of Willis is a network of vascular anastomoses located at the base of the brain, consisting of branches of the internal carotid artery (ICA) and the vertebrobasilar system (VBS). This study evaluated the morphology of the arteries forming the CAC in a sample of Colombian individuals. After cannulation of the ICA and the vertebral artery (VA), the vascular beds of the ACC of 70 brains extracted from cadavers at the Institute of Legal Medicine of Bucaramanga, Colombia, were perfused with polyester resin ( $85 \%$ palatal and $15 \%$ styrene). The anterior communicating artery (ACoA) was observed in 68 brains $(97.1 \%)$, with absence in 2 of the samples $(2.9 \%)$; on average its diameter was $1.91 \pm 1.04 \mathrm{~mm}$ and its length $2.21 \pm 0.97 \mathrm{~mm}$ respectively. Likewise, hypoplasia was found in 6 samples $(8.4 \%)$. The posterior communicating artery (ACoP) was absent in 2 of the samples $(5,7 \%)$; its length was $11.63 \pm 2.12 \mathrm{~mm}$, while its caliber was $1.21 \pm 0.58 \mathrm{~mm}$, being slightly larger on the right side, with no statistically significant differences in relation to the side of presentation $(\mathrm{p}=0.763)$. Hypoplasia of the ACoP was found bilaterally in 19 of the samples (27.1\%) and unilaterally in 15 samples $(21.4 \%)$. Fetal configuration was observed in 8 samples $(20 \%)$ of 35 CACs evaluated. The incidence of ACoP hypoplasia and fetal configuration found in the present study are in the upper segment of those reported in the literature. These morphological expressions have been considered as coadjuvants in the development of cerebrovascular accidents (CVA).

KEY WORDS: Cerebral arterial circle; Posterior Cerebral Artery; Anterior Communicating Artery; Posterior Communicating Artery; Anatomical variations.

\section{REFERENCIAS BIBLIOGRÁFICAS}

Charbel, F. T.; Seyfried, D.; Mehta, B.; Dujovny, M. \& Ausman, J. I. Dominant A1: angiographic and clinical correlations with anterior communicating artery aneurysms. Neurol. Res., 13(4):253-6, 1991.

De Silva, K. R. D.; Silva, R.; Amaratunga, D.; Gunasekera, W. S. L. \& Jayesekera, R. W. Types of the cerebral arterial circle (circle of Willis) in a Sri Lankan population. BMC Neurol., 11:5, 2011.

De Silva, K. R. D.; Silva, T. R. N.; Gunasekera, W. S. L. \& Jayesekera, R. W. Variation in the origin of the posterior cerebral artery in adult Sri Lankans. Neurol. India, 57(1):46-9, 2009.

Eftekhar, B.; Dadmehr, M.; Ansari, S.; Ghodsi, M.; Nazparvar, B. \& Ketabchi, E. Are the distributions of variations of circle of Willis different in different populations? - Results of an anatomical study and review of literature. BMC Neurol., 6:22, 2006.

El Falougy, H.; Weismann, P.; Lukacikova, P.; Mifkovic, A.; Perzelova, A.; Sivakova, I. \& Kubikova, E. The vascular patterns of the posterior part of the circulus arteriosus cerebri (Willisi). Bratisl. Lek. Listy, 119(11):67983, 2018.

Gomes, F. B.; Dujovny, M.; Umansky, F.; Berman, S. K.; Diaz, F. G.; Ausman, J. I.; Mirchandani, H. G. \& Ray, W. J. Microanatomy of the anterior cerebral artery. Surg. Neurol., 26(2):129-41, 1986.

Gunnal, S. A.; Farooqui, M. S. \& Wabale, R. N. Anatomical variability of the posterior communicating artery. Asian J. Neurosurg., 13(2):363-9, 2018.

Hernández-Luna, J.; Casares-Cruz, K.; Rendón-Macías, R. E.; Licea-Medina, D. \& Castillo-Lima, J. A. Evaluación con angiorresonancia magnética nuclear de las variantes anatómicas del círculo arterial cerebral. An. Radiol. Mex., 14:256-61, 2015.
Hoksbergen, A. W. J.; Legemate, D. A.; Csiba, L.; Csáti, G.; Síró, P. \& Fülesdi, B. Absent collateral function of the circle of Willis as risk factor for ischemic stroke. Cerebrovasc. Dis., 16(3):191-8, 2003.

Ingebrigtsen, T.; Morgan, M. K.; Faulder, K.; Ingebrigtsen, L.; Sparr, T. \& Shirmer, $\mathrm{H}$. Bifurcation geometry and the presence of cerebral artery aneurysms. $J$. Neurosurg., 101(1):108-13, 2004.

Kamath, S. Observations on the length and diameter of vessels forming the circle of Willis. J. Anat., 133(Pt. 3):419-23, 1981.

Kapoor, K.; Singh, B. \& Dewan, L. I. J. Variations in the configuration of the circle of Willis. Anat. Sci. Int., 83(2):96-106, 2008.

Kedia, S.; Daisy, S.; Mukherjee, K. K.; Salunke, P.; Srinivasa, R. \& Narain, M. S. Microsurgical anatomy of the anterior cerebral artery in Indian cadavers. Neurol. India, 61(2):117-21, 2013.

Krishnamurthy, A.; Nayak, S. R.; Ganesh Kumar, C.; Jetti, R.; Prabhu, L. V.; Ranade, A. V. \& Rai, R. Morphometry of posterior cerebral artery: embryological and clinical significance. Rom. J. Morphol. Embryol., 49(1):43$5,2008$.

Mandiola, E.; Alarcón, E.; del Sol, M.; Olave, E.; Montero, C.; Sanhueza, P. \& Oñate, J. C. Biometrical analysis of the anterior communicating artery and the anterior cerebral artery in the precommunicating segment of the cerebral arterial circle. Int. J. Morphol., 23(2):171-6, 2005.

Martínez, F.; Spagnuolo Dondero, E.; Calvo Rubal, A.; Laza Núñez, S.; Sgarbi López, N.; Soria Vargas, V. R. \& Prinzo Yamurri, H. Variaciones del sector anterior del polígono de Willis. Correlación anatomoangiográfica y su implicancia n la cirugía de aneurismas intracraneanos. Neurocirugía, 15(6):57889, 2004.

Milenkovic', Z.; Vucetic, R. \& Puzic, M. Asymmetry and anomalies of the circle of willis in fetal brain. Microsurgical study and functional remarks. Surg Neurol., 24(5):563-70,1985.

Monroy-Sosa, A.; Pérez-Cruz, J. C.; Reyes-Soto, G.; Delgado-Hernández, C.; Macías-Duvignau, M. A. \& Delgado-Reyes, L. Importancia de la anatomía microquirúrgica del complejo A1-arteria comunicante anterior. Cir. Cir., 81(4):274-81, 2013.

Pacheco Mayedo, M.; Durán Matos, M. E.; Cuba Yordi, O. L.; Serrano González, L.; Rosales Almeida, Y. \& Loret de Mola Nicolau, J. Patrón común y variantes anatómicas de la porción anterior del círculo arterial del cerebro. Rev. Arch. Med. Camagüey, 21(6), 2017.

Puchades-Orts, A.; Nombela-Gomez, M. \& Ortuño-Pacheco, G. Variation in form of circle of Willis: some anatomical and embryological considerations. Anat. Rec., 185(1):119-23, 1976.

Raghavendra; Shirol, V.; Dixit, D.; Reddy, Y. A. K. \& Desai, S. P. Circle of willis and its variations; morphometric study in adult human cadavers. Int. J. Med. Res. Health Sci., 3(2):394, 2014.

Saeki, N. \& Rhoton Jr., A. L. Microsurgical anatomy of the upper basilar artery and the posterior circle of Willis. J. Neurosurg., 46(5):563-78, 1977.

Siddiqi, H.; Tahir, M. \& Lone, K. P. Variations in cerebral arterial circle of Willis in adult Pakistani population. J. Coll. Physicians Surg. Pak., 23(9):615-9, 2013.

Van Overbeeke, J. J.; Hillen, B. \& Tulleken, C. A. A comparative study of the circle of Willis in fetal and adult life. The configuration of the posterior bifurcation of the posterior communicating artery. J. Anat., 176:45-54, 1991. Wijesinghe, P.; Steinbusch, H. W. M.; Shankar, S. K.; Yasha, T. C. \& De Silva, K. R. D. Circle of Willis abnormalities and their clinical importance in ageing brains: A cadaveric anatomical and pathological study. J. Chem. Neuroanat., 106:101772, 2020

\section{Dirección para correspondencia:}

Dr. Luis E Ballesteros A, MD.

Morfología

Universidad Industrial de Santander

Cra 39 \#51-31

Bucaramanga - COLOMBIA

E-mail: Iballest56@yahoo.es

Recibido : 21-06-2021

Aceptado: 31-07-2021 